\title{
Functional outcomes after resuscitative extracorporeal membrane oxygenation for cardiac arrest: The patients speak for themselves
}

\author{
Joseph M. Bednarczyk, MD, FRCPC, ${ }^{\mathrm{a}, \mathrm{b}}$ and Rohit K. Singal, MD, MSc, FRCSC ${ }^{\mathrm{a}, \mathrm{c}}$
}

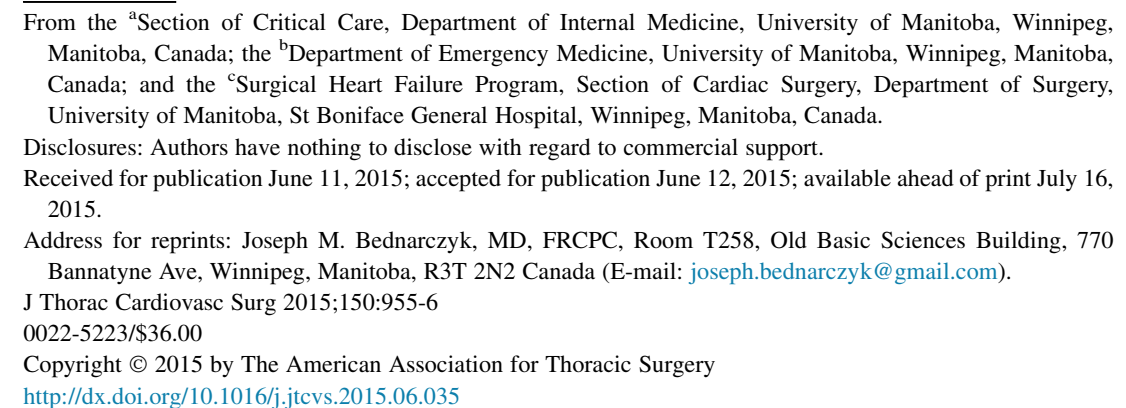

Cardiac arrest remains the most extreme and devastating manifestation of cardiovascular disease. Despite modern approaches to advanced cardiovascular life support, conventional cardiopulmonary resuscitation (CPR) infrequently provides a sufficient therapeutic window to correct the etiology of cardiac arrest. Extracorporeal CPR (E-CPR) describes the emergency application of portable cardiopulmonary bypass during closed-chest compressions to provide a bridge to recovery, decision, or definitive intervention. ${ }^{1}$ Internationally, mounting observational data and propensity-matched analyses of E-CPR have demonstrated a distinct survival advantage relative to conventional CPR in adults with refractory cardiac arrest. ${ }^{2-5}$ The lack of randomized data notwithstanding, E-CPR may represent one of the most important advances in acute resuscitation of the modern era.

Despite the momentum of the current body of E-CPR literature, important concerns warrant consideration. ${ }^{6}$ Although extracorporeal membrane oxygenation (ECMO) may increase short-term survival after prolonged cardiac arrest, it is possible that neurologic injury as a result of sustained hypoperfusion may have long-lasting adverse sequelae. An E-CPR system requires substantial human resources and costs, with reported estimates as high as US $\$ 195,000$ per adult patient surviving to hospital discharge in Taiwan. ${ }^{7}$ Accordingly, robust and long-term clinically relevant outcomes are required to justify significant resource usage and the potential complications of therapy.

In this issue of the Journal, Anselmi and colleagues ${ }^{8}$ report a timely and relevant analysis of a French adult population who did not undergo cardiotomy and had refractory cardiac arrest treated with E-CPR. In this case series, adult patients were eligible for resuscitative ECMO if they had a witnessed episode of cardiac arrest refractory to conventional CPR for at least 30 minutes with a no-flow time not greater than 5 minutes, an end-tidal carbon dioxide level of at least $10 \mathrm{~mm} \mathrm{Hg}$, and a cardiac rhythm other than

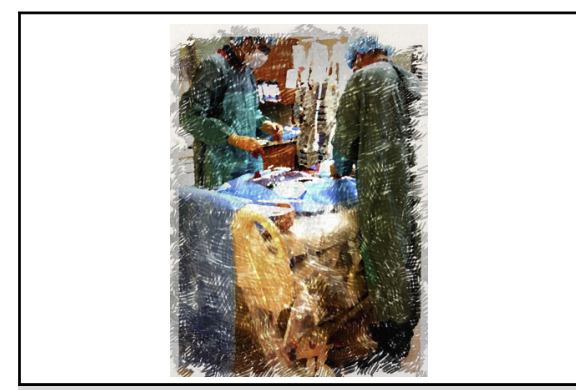

Resuscitative extracorporeal membrane oxygenation cannulation in Winnipeg, Canada (Rohit K. Singal, MD, MSc, FRCSC, and Joseph M. Bednarczyk, MD FRCPC).

\section{Central Message}

Extracorporeal membrane oxygenation cardiopulmonary resuscitation is an emerging therapy for cardiac arrest. Favorable outcomes persist at the duration of longest follow-up in this series by Anselmi and colleagues.

See Article page 947.

asystole. The population included 49 patients, of whom $43(88 \%)$ sustained in-hospital cardiac arrest and 6 $(12 \%)$ sustained out-of-hospital cardiac arrest. The cause of hemodynamic collapse was a primary cardiac etiology in the majority of cases (30 [61\%]), followed by trauma, acute respiratory illness, and other etiologies. The mean low-flow duration was $47.2 \pm 33$ minutes, and the mean blood lactate level was $11.7 \pm 6 \mathrm{mmol} / \mathrm{L}$, illustrating the extreme burden of illness in this cohort.

Several technical aspects of Anselmi and colleagues' E-CPR protocol are noteworthy. ${ }^{8}$ First, cannulation was performed through a modified cutdown to expose the femoral vessels, an approach that they considered to maximize efficiency. This differs from the percutaneous Seldinger approach described in recent reports in Australia and Canada. ${ }^{9,10}$ Second, $6 \mathrm{~F}$ to $10 \mathrm{~F}$ distal perfusion cannulas were directed to the superficial femoral artery in the lower extremities with arterial ECMO cannulas, a safety measure not routinely performed in all centers. Impressively, only 1 patient in the series had extremity ischemia develop. Finally, in the latter portion of the series, patients who had excessive left ventricular afterload on ECMO were treated with minimally invasive approaches, such as intra-aortic balloon counterpulsation or transaortic axial flow pumps (Impella Abiomed Inc, 
Aachen, Germany), rather than proceeding to cardiotomy and left ventricular vent insertion.

The most provocative aspect of this report is the unique set of outcomes reported. Anselmi and colleagues ${ }^{8}$ demonstrated that 18 patients $(36.7 \%)$ survived to hospital discharge, with no patients dying during an extended follow-up interval of $15.6 \pm 19.2$ months. Patients who had survived to discharge were contacted by telephone to complete the Short-Form (36) Health Survey to assess their overall quality of life and functional status. Compared with age- and sex-matched control subjects from the French population, survivors displayed similar scores in the majority of domains, including mental health, social activity, general health, and physical pain, although role limitation and physical activity scores were comparatively reduced. This suggests that despite the prolonged hospital course endured by patients $(79.1 \pm 97.6$ days) and the occasional requirement for advanced therapy, including cardiac transplantation or long-term ventricular assist device placement, patients had stable long-term survival with acceptable functional status and overall quality of life after E-CPR.

This observational report is subject to several common limitations of research in the field of extracorporeal resuscitation, including confounding by indication. ${ }^{11}$ The case series contained a small sample size and lacked randomization or a propensity-matched control group. This study may have been particularly susceptible to selection bias because of its relatively extensive list of inclusion and exclusion criteria and the incorporation into selection of physician judgment surrounding overall comorbidities and life expectancy. Of note, the mean age of patients in this study ( $47.6 \pm 16$ years) was somewhat lower than in recent reports, ${ }^{9,12,13}$ and younger age is a known predictor of favorable outcome in this population. ${ }^{14}$

As Anselmi and colleagues ${ }^{8}$ imply, success across centers will vary according to case selection and program organization. For centers developing or refining E-CPR programs, observational data gleaned from reports such as Anselmi and colleagues ${ }^{8}$ are crucial, because randomized trials of E-CPR are largely considered unethical as a result of the lack of clinical equipoise. ${ }^{15}$ Their clear protocol description and attention to clinically relevant, patient-centered, and long-term outcomes is a fine example of the reporting needed to advance the field. Standardization of E-CPR outcome reporting to include long-term complications, hospital readmissions, and return to personal and societal productivity will better inform us of the potential benefits offered to patients. This article by Anselmi and colleagues ${ }^{8}$ is a strong contribution to this effort.

\section{References}

1. Shinar Z, Bellezzo J, Paradis N, Dembitsky W, Jaski B, Mallon W, et al. Emergency department initiation of cardiopulmonary bypass: a case report and review of the literature. J Emerg Med. 2012;43:83-6.

2. Shin TG, Choi JH, Jo IJ, Sim MS, Song HG, Jeong YK, et al. Extracorporeal cardiopulmonary resuscitation in patients with in-hospital cardiac arrest: a comparison with conventional cardiopulmonary resuscitation. Crit Care Med. 2011;39:1-7.

3. Shin TG, Jo IJ, Sim MS, Song YB, Yang JH, Hahn JY, et al. Two-year survival and neurological outcome of in-hospital cardiac arrest patients rescued by extracorporeal cardiopulmonary resuscitation. Int J Cardiol. 2013;168:3424-30.

4. Maekawa K, Tanno K, Hase M, Mori K, Asai Y. Extracorporeal cardiopulmonary resuscitation for patients with out-of-hospital cardiac arrest of cardiac origin: a propensity-matched study and predictor analysis. Crit Care Med. 2013;41:1186-96.

5. Chen YS, Lin JW, Yu HY, Ko WJ, Jerng JS, Chang WT, et al. Cardiopulmonary resuscitation with assisted extracorporeal life-support versus conventional cardiopulmonary resuscitation in adults with in-hospital cardiac arrest: an observational study and propensity analysis. Lancet. 2008;372:554-61.

6. Lyon RM. Extra-corporeal cardiopulmonary resuscitation-miracle cure or expensive futility? Resuscitation. 2012;83:1311-2.

7. Chen YS. Organization of ECPR rescue team. In: International Course on ECMO and Euro-ELSO. Available at: http://www.paris-ecmo.org/presentations/2014/ presentations/ppt/in118/index.html. Accessed June 11, 2015.

8. Anselmi A, Flécher E, Corbineau H, Langanay T, Le Bouquin V, Bedossa M, et al. Survival and quality-of-life after extracorporeal life support for refractory cardiac arrest: a case series. J Thorac Cardiovasc Surg. 2015;150:947-54.

9. Bednarczyk JM, White CW, Ducas RA, Golian M, Nepomuceno R, Hiebert B, et al. Resuscitative extracorporeal membrane oxygenation for in hospital cardiac arrest: a Canadian observational experience. Resuscitation. 2014;85: 1713-9.

10. Stub D, Bernard S, Pellegrino V, Smith K, Walker T, Sheldrake J, et al. Refractory cardiac arrest treated with mechanical CPR, hypothermia, ECMO and early reperfusion (the CHEER trial). Resuscitation. 2015;86:88-94.

11. Kilbaugh TJ, Srinivasan V, Berg RA, Nadkarni VM. Propensity, prophecy, and perplexity: does in-hospital extracorporeal cardiopulmonary resuscitation really make a difference? Resuscitation. 2010;81:786-7.

12. Lin JW, Wang MJ, Yu HY, Wang CH, Chang WT, Jerng JS, et al. Comparing the survival between extracorporeal rescue and conventional resuscitation in adult in-hospital cardiac arrests: propensity analysis of three-year data. Resuscitation. 2010;81:796-803.

13. Haneya A, Philipp A, Diez C, Schopka S, Bein T, Zimmermann M, et al. A 5-year experience with cardiopulmonary resuscitation using extracorporeal life support in non-postcardiotomy patients with cardiac arrest. Resuscitation. 2012;83: 1331-7.

14. Mendiratta P, Wei JY, Gomez A, Podrazik P, Riggs AT, Rycus P, et al. Cardiopulmonary resuscitation requiring extracorporeal membrane oxygenation in the elderly: a review of the Extracorporeal Life Support Organization registry. ASAIO J. 2013;59:211-5.

15. Bartlett RH. Clinical research in acute fatal illness: lessons from extracorporeal membrane oxygenation. J Intensive Care Med. September 15, 2014 [Epub ahead of print]. 